\title{
Last call for the flight simulation test?
}

\author{
Luke S. Howard \\ Affiliation: National Pulmonary Hypertension Service, Hammersmith Hospital, Imperial College Healthcare \\ NHS Trust, London, UK.
}

Correspondence: L.S. Howard, National Pulmonary Hypertension Service, Hammersmith Hospital, Imperial College Healthcare NHS Trust, Du Cane Road, London, W12 OHS, UK. E-mail: L.howarddimperial.ac.uk

0 @ERSpublications

Measuring $\mathrm{PO}_{2}$ during flight simulation fails to predict which COPD patients develop complications during air travel http://ow.ly/nQ1Xo

\begin{abstract}
"Dear Colleague, Please see and advise on this patient with COPD who wishes to fly". This is a common request from our non-respiratory colleagues and although we have guidelines to follow to assist in answering their queries, the evidence on which the guidelines are based is weak. The most recently published air travel recommendations are those from the British Thoracic Society (BTS) [1], which try to make some sense of the evidence and provide guidance based on expert consensus, but the recommendations for prescribing oxygen during a flight remain, at best, level C.
\end{abstract}

Guidance has therefore had to remain vague in terms of which patients with chronic obstructive pulmonary disease (COPD) should be assessed for in-flight oxygen: those with previous respiratory symptoms during air travel, severe COPD (forced expiratory volume in $1 \mathrm{~s}<30 \%$ predicted) or bullous lung disease. Once these patients have been identified, there is still uncertainty on how to assess them further and the physician can chose between a simple 50-m walk test, using equations to predict the need for oxygen based on predicted partial pressure of oxygen $\left(\mathrm{PO}_{2}\right)$ at cabin pressure, or by undertaking a formal high altitude simulation test (HAST), all of which are reviewed in detail in the BTS recommendations [1].

The first test assumes that if a patient can walk $50 \mathrm{~m}$ then this indicates sufficient cardiopulmonary reserve to withstand a fall in inspired $\mathrm{PO}_{2}$ (to the equivalent of approximately $15 \%$ inspired oxygen at sea level) when flying in commercial aircraft. The second two methods assume that a resulting arterial $\mathrm{PO}_{2}\left(\mathrm{PaO}_{2}\right)$ $<6.6 \mathrm{kPa}(50 \mathrm{mmHg})$ is a reliable indicator of the need for in-flight oxygen. This cut-off is based on expert consensus [2] in the absence of evidence to support any particular value. Since the body can tolerate far lower degrees of hypoxaemia [3], there is no physiological measure to otherwise determine a suitable cutoff. In the current issue of the European Respiratory Journal (ERJ), EDVARDSEN et al. [4] further challenge the usefulness of the HAST.

It seems universally accepted, even by the most sceptical [5], that patients with COPD with daytime hypoxaemia already receiving oxygen at sea level should receive supplementary oxygen during a flight. Those patients aside, in trying to assess a patient who does not normally receive oxygen, simulating the cabin environment would seem an appropriate method to achieve this, but having established how to do the test, what should be its output? Measuring $\mathrm{PaO}_{2}$ during a HAST is easy enough to do, albeit variably unpleasant for the patient, but it would only be useful if it were to predict outcomes. Levels of hypoxaemia dangerous enough to cause life-threatening organ dysfunction in patients without major cardiac comorbidity, such as myocardial ischaemia or arrhythmia, are physiologically inconceivable in patients who do not need oxygen at sea level [3], so what is it we are actually trying to predict and thus prevent?

Respiratory symptoms during flights are relatively common in studies of patients with COPD, with varying frequencies being reported, most likely depending on the method of recruitment. The UK Flight Outcomes Study [6] was a prospective study in which 243 patients with COPD took part (Global Initiative for Chronic

Received: March 012013 | Accepted: March 052013

Conflict of interest: None declared.

Copyright (C)ER 2013 
Obstructive Lung Disease severity: mild 2\%; moderate 29\%; severe 43\%; very severe 26\%), of whom $18 \%$ reported some form of respiratory distress, usually mild. Another large study of 391 patients, this time retrospective and from the same group who published the current study, reported a $25 \%$ incidence of respiratory symptoms in COPD patients, with symptoms more likely to occur in patients with more severe breathlessness at sea level [7]. Mild symptoms are likely to be of little consequence, but more severe symptoms may be distressing, necessitate medical attention and possibly even flight diversion.

The authors in the latter study used the term "hypoxia-related symptoms" to refer to symptoms such as air hunger and dyspnoea, although they did not show that these patients were more hypoxaemic or that they related to hypoxaemia through correlation. While this may seem picky, breathlessness itself is not caused by hypoxaemia: indeed, their current study shows that in-flight symptoms were not related to hypoxaemia during the HAST. While hypoxaemia does increase ventilatory drive, at the levels likely to be seen during a flight, this will be modest [8], thus it is those patients with more precarious respiratory mechanics who are most likely to be affected, rather than just those with lower $\mathrm{PaO}_{2}$ as a result of the HAST or decompression in an aircraft.

In the current issue of the ERJ, EDVARDSEN et al. [7] report on a group of 82 patients with COPD who had all recently flown and were referred for HAST. $46 \%$ had experienced in-flight respiratory symptoms, suggesting an enriched population. A surprisingly high proportion $(n=14(17 \%))$ had required medical attention within $48 \mathrm{~h}$ of landing, half of which required hospitalisation. All 14 patients requiring medical attention had a $\mathrm{PaO}_{2}<6.6 \mathrm{kPa}$ on the subsequent HAST and the mean $\mathrm{PaO}_{2}$ of those with respiratory symptoms during the HAST was $6.3 \mathrm{kPa}$. This might imply that the HAST could predict poor outcomes during flights; however, patients who travelled without symptoms had the same $\mathrm{PaO}_{2}$ of $6.3 \mathrm{kPa}$ during the HAST, suggesting otherwise.

Although hypoxaemia during HAST could not predict who would develop symptoms, the authors showed that when in-flight oxygen was used on subsequent flights, there was a reduction in symptoms. EDVARDSEN et al. [7] treat this as a contradictory finding, suggesting that the uncontrolled nature of the intervention may have led to psychological benefits. This may not be the case, rather it could be seen as proof of the hypothesis that symptoms are not in fact directly related to hypoxaemia. Put simply, the hypoxic stimulus is similar in both groups, with and without symptoms, but what may differ is the respiratory mechanical load in response to hypoxaemia, such that when the stimulus is removed with in-flight oxygen symptoms abate.

It would have been neater had those who had experienced symptoms during flights also experienced worse breathlessness during the HAST, but this was not the case. This remains unexplained other than the fact that the HAST is much shorter than the flight and involves no form of exertion.

Where does this leave us? More attempts to develop algorithms and equations to predict the $\mathrm{Pa}_{2} \mathrm{O}_{2}$ in place of a HAST would seem misplaced, as would studies to find a better cut-off from the HAST. It would appear that functional status at sea level may be a better indicator of the need for oxygen, but which measurement to use is by no means clear. As the study by EDVARDSEN et al. [7] would suggest, one plausible candidate might be the Medial Research Council breathlessness scale, since this was associated with in-flight symptoms. The BTS recommendations have taken this approach for many of the cardiac conditions, e.g. recommending in-flight oxygen for all heart failure patients in New York Heart Association functional class IV and all patients with pulmonary hypertension who suffer functional class III-IV symptoms [1]. This has, however, come in for fair criticism since it is non-evidence-based, but perhaps no less so than the HAST, and results in a high number of possibly unnecessary prescriptions [9].

Using exercise as a measure of pulmonary reserve is attractive, since it provides an alternative form of increased respiratory drive, is simple to undertake and can be standardised. EDVARDSEN et al. [10] have also recently published a further study using exercise to predict the need for in-flight oxygen, but using HAST as the gold standard. Once again, knowing what parameter to measure on exercise requires further investigation and looking beyond saturation to exercise capacity and symptoms is likely to be more physiologically relevant. Furthermore, there is no good reason to believe why exercising in ambient conditions at sea level would be significantly inferior to exercising at simulated altitude, if one is simply trying to test pulmonary reserve. It would also be much simpler.

What is needed is a better understanding of the associations between baseline sea-level physiology and inflight symptoms, followed up by a well-designed study to show that intervention is effective. It has been 13 years since NAEIJE [5] called for proponents of the HAST to design a study showing that it is relevant and effective. The current study suggests a new approach is required. Hypoxaemia is not the cause of hypoxiarelated symptoms. 


\section{References}

1 Ahmedzai S, Balfour-Lynn IM, Bewick T, et al. Managing passengers with stable respiratory disease planning air travel: British Thoracic Society recommendations. Thorax 2011; 66: Suppl. 1, i1-i30.

2 Gong H Jr, Tashkin DP, Lee EY, et al. Hypoxia-altitude simulation test. Evaluation of patients with chronic airway obstruction. Am Rev Respir Dis 1984; 130: 980-986.

Bartsch P, Gibbs JS. Effect of altitude on the heart and the lungs. Circulation 2007; 116: 2191-2202.

Edvardsen A, Ryg M, Akerø A, et al. COPD and air travel: does hypoxia-altitude simulation testing predict in-flight respiratory symptoms? Eur Respir J 2013; 42: 1216-1223.

Naeije R. Preflight medical screening of patients. Eur Respir J 2000; 16: 197-199.

Coker RK, Shiner RJ, Partridge MR. Is air travel safe for those with lung disease? Eur Respir J 2007; 30: 1057-1063.

Edvardsen A, Akero A, Hardie JA, et al. High prevalence of respiratory symptoms during air travel in patients with COPD. Respir Med 2011; 105: 50-56.

8 Cunningham DJC, Robbins PA, Wolff CB. Integration of respiratory responses to changes in alveolar partial pressures of $\mathrm{CO}_{2}$ and $\mathrm{O}_{2}$ and in arterial pH. In: Fishman AP, Cherniack NS, Widdicombe JG, eds. Handbook of Physiology: The Respiratory System. Bethesda, American Physiological Society, 1986: pp. 475-528.

9 Burns RM, Peacock AJ, Johnson MK, et al. Hypoxaemia in patients with pulmonary arterial hypertension during simulated air travel. Respir Med 2013; 107: 298-304.

10 Edvardsen A, Akero A, Christensen CC, et al. Air travel and chronic obstructive pulmonary disease: a new algorithm for pre-flight evaluation. Thorax 2012; 67: 964-969. 